\title{
Thermal impedance plots of micro-scaled devices
}

\author{
B. Vermeersch, G. De Mey* \\ Department of Electronics and Information Systems (ELIS), University of Ghent, Sint Pietersnieuwstraat 41, 9000 Ghent, Belgium
}

Received 21 February 2005; received in revised form 2 May 2005

Available online 5 July 2005

\begin{abstract}
The complex thermal impedance $Z_{\text {th }}$ of a microelectronic heat source on the surface of a silicon wafer has been calculated semi-analytically as a function of the frequency. By representing the results in a Nyquist plot, almost perfect circular curves are obtained. This result is analogous to complex loci of the dielectric constant obtained for some materials.
\end{abstract}

(c) 2005 Elsevier Ltd. All rights reserved.

\section{Introduction}

In present day electronics and microelectronics, thermal problems are found to become more dominant due to the high power densities. The latter being proportional to the clock frequency (for a digital circuit), it is expected that thermal problems will need more and more attention in the future.

Usually the high operating temperature of an integrated circuit is considered as the only thermal problem. However, even if the total dissipated power is quite moderate, the temperature distribution can still give rise to malfunctioning circuits. The reason is quite simple: all transistors have temperature dependent characteristics. Even a temperature fluctuation of a few degrees may have an influence on the electric behaviour of the circuit. It must be emphasised here that this problem is not limited to uniform heating of a chip but is much more important for non-uniform and time dependent heating.

\footnotetext{
* Corresponding author. Tel.: +32926433 86; fax: +32 9264 8961.

E-mail addresses: bjorn.vermeersch@ugent.be (B. Vermeersch), demey@elis.ugent.be (G. De Mey).
}

Unwanted couplings between different parts of a circuit can be induced thermally.

Therefore, the need arises not to limit one self to the study of the thermal resistance $R_{\mathrm{th}}$ but also to the thermal impedance $Z_{\text {th }}$ of a transistor in order to deal with time or frequency dependent problems.

In this paper we limit ourselves to a linear thermal analysis. Hence the temperature dependence of the thermal conductivity $k$ or other thermal parameters has not been taken into account. Otherwise, the thermal conduction problem becomes non-linear and strictly speaking a thermal impedance cannot be defined. Experimentally, thermal impedance measurements are always performed at a maximum junction temperature (above ambient) of less than $20^{\circ} \mathrm{C}[1]$.

The originality of the present research is proved by the fact that only a few papers on related topics could be found in the literature [2]. The most interesting result is that the impedance plot turned out to be circular graphs. As a consequence, the AC thermal behaviour is characterised by a limited number of parameters (centre and radius of the circle). For the point of view of reliability it might be useful to measure these parameters as a function of time in order to detect the reliability in the close neighbourhood of the heat sources. 
Although a very limited number of papers also displayed circular Nyquist plots, no one pointed out the perfect analogy with the theory of dielectrics published by Fuoss and Kirkwood long time ago [3].

\section{Calculation of the thermal impedance $Z_{\text {th }}$}

Due to the small dimensions of the heat sources involved in microelectronics one can consider the semiconductor substrate as a half infinite volume $(z<0)$ with a heat source on the top surface $z=0$ (Fig. 1).

In phasor notation the temperature distribution $T(x, y, z)$ in the substrate satisfies the equation [4]:

$k \nabla^{2} T(x, y, z)-\mathrm{j} \omega C_{v} T(x, y, z)=0$,

where $k$ is the thermal conductivity (in $\mathrm{Wm}^{-1} \mathrm{~K}^{-1}$ ), $C_{v}$ the thermal capacity per unit volume (in $\mathrm{J} \mathrm{m}^{-3} \mathrm{~K}^{-1}$ ), $\mathrm{j}=\sqrt{-1}$ the imaginary unit and $\omega=2 \pi f$ the angular frequency (in $\mathrm{Hz}$ ).

For a point shaped heat source dissipating a power $P$ (in $\mathrm{W}$ ) and located on the surface $z=0$ in the point $\left(x^{\prime}, y^{\prime}, 0\right)$, the temperature distribution is given by:

$T(x, y, z)=\frac{P}{2 \pi k r} \exp \left(-\sqrt{\frac{\mathrm{j} \omega C_{v}}{k} r}\right)$,

where $r$ is the distance between the field point $(x, y, z)$ and the source point $\left(x^{\prime}, y^{\prime}, 0\right)$. The square root of $j$ must be chosen as $\sqrt{\mathrm{j}}=\frac{\sqrt{2}}{2}(1+j)$ in order to ensure convergence for $r \rightarrow \infty$.

If the power $P$ is omitted and the factor 2 in the denominator of (2) is replaced by 4 , one will recognise the Green's function or the fundamental solution of Eq. (1) in the three-dimensional space. The factor 2 is due to the fact that the semiconductor material occupies only the half space $z<0$.

On the surface $z=0$ the adiabatic boundary condition has been assumed. The thermal conductivity $k=160 \mathrm{~W} \mathrm{~m}^{-1} \mathrm{~K}^{-1}$ of silicon is relatively high with respect to the value $k=0.025 \mathrm{Wm}^{-1} \mathrm{~K}^{-1}$ of the dry air fill-

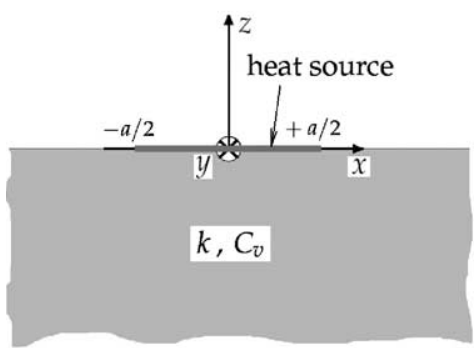

(a) cross sectional view ing $(z>0)$ in an electronic package. Taking this into account, the adiabatic condition is sufficiently accurate. Although this boundary condition may be questionable for the DC analysis of the electronic package, for time dependent or AC problems it is sufficient to consider only the temperature distribution inside the silicon. We have verified from our calculations that the AC temperature field is completely decayed (i.e., $|T|<10^{-3}|T|_{\max }$ ) at a distance of $100 \mu \mathrm{m}$ from the heat source for frequencies $f>100 \mathrm{kHz}$.

For a rectangular heat source with dimensions $a$ and $b$ (Fig. 1), and a power dissipation $P$ or a power density $p=\frac{P}{a b}$ (in $\mathrm{W} / \mathrm{m}^{2}$ ), the temperature distribution can be found by superposition:

$$
\begin{aligned}
T(x, y, z)= & \frac{p}{2 \pi k} \int_{-a / 2}^{a / 2} \mathrm{~d} x^{\prime} \int_{-b / 2}^{b / 2} \mathrm{~d} y^{\prime} \\
& \times \frac{\exp \left(-\sqrt{\frac{j \omega C_{v}}{k}} \sqrt{\left(x-x^{\prime}\right)^{2}+\left(y-y^{\prime}\right)^{2}+z^{2}}\right)}{\sqrt{\left(x-x^{\prime}\right)^{2}+\left(y-y^{\prime}\right)^{2}+z^{2}}} .
\end{aligned}
$$

The integral (3) has been evaluated numerically by dividing the heat source area into $N$ small sub areas. The numerical convergence was checked by using different values of $N$. The singularity in the integrand of (3), which occurs for $x=x^{\prime}, y=y^{\prime}$ and $z=0$, has been calculated separately by an exact analytical integration [5].

Strictly speaking, the thermal impedance is defined as the temperature difference between a heat source (assumed at a uniform temperature) and the temperature of a heat sink (here $T=0$ at infinity) divided by the heat flow. In our situation the temperature of the heat source is not uniform at all. Hence the impedance has been evaluated by taking (i) the average temperature and (ii) the maximum temperature (i.e. the maximum absolute value) of the heat source evaluated by (3).

A Nyquist plot of both kinds of impedances is shown in Fig. 2.

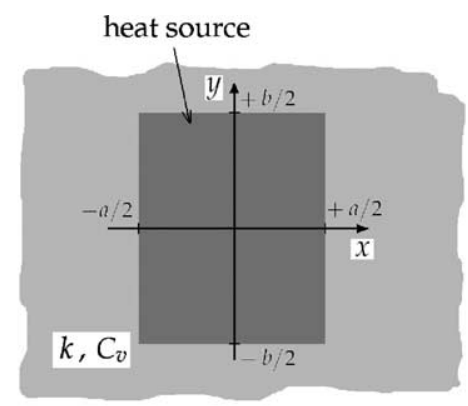

(b) top view

Fig. 1. Rectangular heat source on top of half infinite thermal conductor $(z<0)$. 


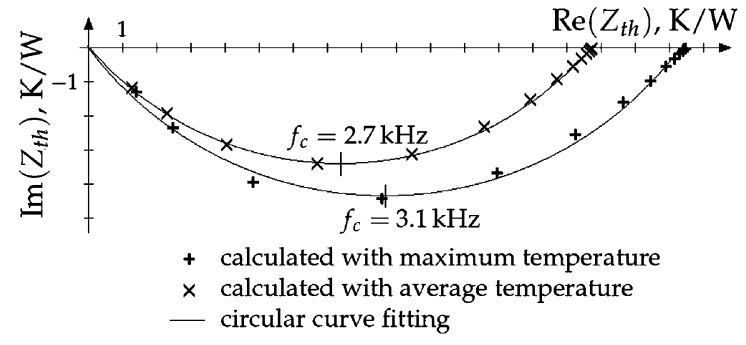

Fig. 2. Nyquist plot of thermal impedance $Z_{\mathrm{th}}$.

These results have been obtained for the thermal parameters of $\mathrm{Si}\left(k=160 \mathrm{Wm}^{-1} \mathrm{~K}^{-1}\right.$ and $C_{v}=1.784 \times$ $10^{6} \mathrm{~J} \mathrm{~m}^{-3} \mathrm{~K}^{-1}$ ) and a square shaped heat source of $200 \mu \mathrm{m} \times 200 \mu \mathrm{m}$. However, by rearranging (3) in a dimensionless form, it can be easily proved that those parameters have no influence on the shape of the Nyquist plot. Just the frequency scale has to be adjusted if smaller heat sources or another semiconductor material is investigated.

The same kind of Nyquist plots have been obtained for rectangular heat sources with aspect ratios $a / b$ up to 10 . The central frequency $f_{\mathrm{c}}$, where the imaginary part of $Z_{\text {th }}$ reaches its minimum value, has also been determined. From all these data the following relationship was found by curve fitting:

$f_{\mathrm{c}}=1.20 \frac{1}{a b} \frac{k}{C_{v}}$.

It has been verified that (4) can even be applied with an accuracy of $10 \%$ for aspect ratios alb up to 48 .

A more careful inspection of both curves in Fig. 2 reveals that a curve fitting with a circle intersecting the horizontal axis at $45^{\circ}$, is almost perfect. Although it can be proved mathematically that (3) does not give rise to an exact circular plot. The particular form of the $\mathrm{Ny}$ quist curve corresponds with the experimental measurements performed on electronic packages. The high frequency part of these results were found to be a circle with the same intersection angles $[1,6]$.

Experimentally, the temperature of a microcircuit at chip level is usually measured by using sensor diodes or any other junction. These devices deliver a signal proportional to the average device temperature. Hence, the use of the impedance using the average temperature is more sound (Fig. 2).

\section{Discussion}

The most remarkable fact of the Nyquist plot of Fig. 2 is the almost perfect circular shape. Similar results have been reported in the literature many years ago about the complex dielectric constant of some materials
$[7,8]$. A Nyquist plot of this quantity is called the ColeCole plot.

The explanation for this phenomenon was the existence of a Debye relaxation mechanism in the dielectric layer. If one single time constant was taken into account, the Nyquist plot of the complex dielectric constant turned out to be a circle with its centre point located on the real axis. Experimentally the Nyquist plots were indeed circles, but with their central point above the real axis just like the plot of Fig. 2. This aspect has been explained successfully by introducing a probability distribution for the relaxation time constants [3].

For the thermal impedance plot on the other hand, similar plots have been obtained although no probability distribution has been used. All parameters like $k$, $C_{v}$ were kept constant. It proves that a homogeneous thermal conductor involves a spectrum of thermal time constants rather than a single value. This conclusion also agrees with the experimental results published by Székely et al. obtained from transient temperature measurements on electronic packages [9-11].

\section{Conclusion}

The Nyquist plot of the thermal impedance of a rectangular heat source on a half infinite thermal conductor has been evaluated numerically. All the Nyquist plots show a circular curve just like the Cole-Cole plots observed for dielectric layers involving Debye relaxation mechanism. Hence, the theories developed for dielectric layers can be directly applied to the AC thermal impedance analysis, for example to determine the time constant distribution.

\section{References}

[1] Kawka P. Thermal impedance measurements and dynamic modelling of electronic packages. $\mathrm{PhD}$ thesis, University of Ghent, 2005.

[2] Székely V. Identification of RC networks by deconvolution: chances and limits. IEEE Trans Circuits Syst-I: Fund Theor Appl 1998;45(3):244-58.

[3] Fuoss RM, Kirkwood JG. Electrical properties of solids: VIII dipole moments in polyvinyl chloride-diphenyl systems. J Am Chem Soc 1941;63:385-94.

[4] De Mey G. Various applications of the boundary element method. Oradea: Ed. University of Oradea, Romania; 2002.

[5] Dean DJ. Thermal design of electronic circuit boards and packages. Ayr, Scotland: Electrochemical publications; 1985.

[6] Kawka P, De Mey G, Napieralski A. Electronic package thermal description by dielectric polarization theory. In: Proceedings of the 11th MIXDES conference 2004, June 24-26, Szczecin, Poland. p. 287-90. 
[7] Cole KS, Cole RH. Dispersion and absorption in dielectrics: I-alternating current characteristics. J Chem Phys 1949;9:341-51.

[8] Jonscher AK. Dielectric relaxation in solids. J Phys D: Appl Phys 1999;32:R57-70.

[9] Székely V, Van Bien T. Fine structure of heat flow path in semiconductor devices: a measurement and identification method. Solid-State Electron 1988;31:1363-8.
[10] Székely V. Thermal monitoring of microelectronic structures. Microelectron J 1994;25(3):157-70.

[11] Székely V, Rencz M. Thermal investigations of microsystems. In: Proceedings of NATO advanced research workshop on multichip models with integrated sensors, May 18-20, 1995, Budapest, Hungary. p. 1-10. 